\title{
Rotigotine as Pharmacological Augmentation Strategy of Early Rehabilitation of Acute Ischemic Stroke Patient
}

\author{
Sabrina Anticoli ${ }^{*}$, Maria Cristina Bravi ${ }^{1}$, Antonio Siniscalchi ${ }^{2}$ and Francesca Romana Pezzella ${ }^{1}$ \\ ${ }^{1}$ Emergency Department, Azienda Ospedaliera San. Camillo-Forlanini, Circonvallazione Gianicolense, Rome \\ ${ }^{2}$ Department of Neurology, Annunziata Hospital , Via F. Migliori, Cosenza, Italy
}

\section{Letter to Editor}

Early rehabilitation in stroke patient is generally regarded as key strategy to improve clinical outcome and patient's quality of life. Based on theoretical considerations of brain plasticity and experimental studies pharmacological augmentation of stroke rehabilitation might be a reasonable intervention to further improve stroke patient prognosis. Rotigotine with transdermal delivery system continuous over $24 \mathrm{hrs}$ that provides an exciting and important opportunity to manipulate the brain's pharmacological environment at a time when physiological remodeling of the brain is occurring through conventional rehabilitation treatments [1]. Understanding the relationship between pharmacologically primed neuroplasticity and practice dependent neuroplasticity is of major scientific interest in understanding how the brain adapts to injury. Some previous studies have suggested that levodopa in combination with physiotherapy may have a positive effect on motor deficits following stroke $[2,3]$.

The aim of this study is to evaluate the functional outcome and the possible adverse events in early start (within $24 \mathrm{hr}$ on stroke unit admission) treatment of rotigotine patch [1] on motor deficits following stroke of middle cerebral artery of both hemisphere. We summarize here our experience with rotigotine patches $2 \mathrm{mg} / 24 \mathrm{hrs}$ as pharmacological augmentation strategy in early rehabilitation of acute ischemic stroke patient on motor recovery after stroke. We included 40 consecutive patients with acute stroke confirmed by Computerized Tomography (CT) scan and MRI. All patients provided written informed consent before participating to the observation.

We limited the observation to a small group of patients with acute ischemic stroke resulting in a severe hemiparesis/hemiplegia (NIHSS score on motor item range 3-4) with premorbid mRankin $=0$. Exclusion criteria were as follows: A pre-existing neurological condition (e.g., dementia, Parkinson's disease, multiple sclerosis) that would confound cognitive or motor assessments; acute concomitant illness (e.g., infection, unstable angina, myocardial infarction or heart, respiratory, renal or liver failure); systolic blood pressure $<120 \mathrm{mmHg}$ and/or diastolic $<70 \mathrm{mmHg}$ (as dopamine agonists may lead to postural hypotension, especially during dose escalation); exposure to any other investigational drug within 30 days of enrollment in the study; presence of clinically significant drug or alcohol abuse within the previous 6 months; pregnancy and no r-TPA treatment.

Each patient received $30 \mathrm{~min}$ of rehabilitation twice a day within $24 \mathrm{hrs}$ stroke onset and stroke unit standard care. Treatment with rotigotine patches $2 \mathrm{mg}$ daily was started within $24 \mathrm{hrs}$ stroke onset as well. We included 40 consecutive patients, with acute stroke confirmed by CT scan and MRI. Sixteen patients were treated with rotigotine and physiotherapy while 24 patients were treated only with physiotherapy. The groups were homogeneous by gender, age and NIHSS score. In the rotigotine group were selected 16 patients ( $12 \mathrm{~F}, 4 \mathrm{M})$ mean age $79.66 \pm 5.97$, NIHSS score on admittance to the stroke unit was $16.20 \pm 4.79$, with motor items score being 3 or 4 . In the not-Rotigotine group were selected
24 patients $(15 \mathrm{~F}, 9 \mathrm{M})$ media age $79.26 \pm 9.19$, mean NIHSS score on admittance to the stroke unit was $15.04 \pm 4.30$ with motor item score being 2 or 4 . After seven days, in the Rotigotine group, global mean NIHSS score was $12.20 \pm 4.22$ with more than $50 \%$ (11 patients) showing an improvement of motor item score $(\mathrm{P}<0.05$ using paired Student $\mathrm{t}$-Test). In the not-Rotigotine group global mean NIHSS score was $13.08 \pm 4.43 ; 9$ patients showed an improvement of motor item score $(\mathrm{P}=\mathrm{NS})$. There were no serious adverse events using the patch that was well tolerated. The increase in motor outcome was significantly higher in Rotigotine-group than not Rogotine group that were treated only with physiotherapy.

Previously, Gorgoraptis et al. reported that no significant improvement in motor performance was observed in poststroke patients in treatment with rotigotine without physiotherapy. Furthermore, in this study the patients were eligible only if stroke onset was at least 9 days before the first assessment session [4]. Based on our experience rotigotine in combination with early physiotherapy may enhances motor recovery after stroke. The final evaluation was carried out after only six days from the ischemic event in agreement with the time of hospitalization in the Stroke Unit. It was used the NHISS because is of easy administration by the same personnel of Stroke Unit. The scale of mRankin which assesses the overall functional capacity of the patient is not valid in identifying small changes in motor skills. The limits of the study are the small number of the sample; do not have been carried out in blind and not to be a randomized trial. Further randomized and blind study, using more sensitive scales such us the Fugl-Meyer scale and a follow-up of the patients are needed to confirm these data.

\section{References}

1. Grosset D (2006) The Rotigotine Transdermal Patch May Provide Continuous Dopaminergic Stimulation in Early-Stage Parkinson's Disease. Adv Clin Neurol Rehab 6: 32-34.

2. Scheidtmann K, Fries W, Müller F, Koenig E (2001) Effect of levodopa in combination with physiotherapy on functional motor recovery after stroke: a prospective, randomised, double-blind study. Lancet 358: 787-790.

3. Scheidtmann K (2004) Advances in adjuvant pharmacotherapy for motor rehabilitation: effects of levodopa. Restor Neurol Neurosci 22: 393-398.

4. Gorgoraptis N, Mah YH, Machner B, Singh-Curry V, Malhotra P, et al. (2012) The effects of the dopamine agonist rotigotine on hemispatial neglect following stroke. Brain 135: 2478-2491.

${ }^{*}$ Corresponding authors: Sabina Anticoli, Stroke Unit, Emergency Department Azienda Ospedaliera S. Camillo-Forlanini, Circonvallazione Gianicolense, Rome, Italy, Tel: +390658703313; E-mail: sabrina.anticoli@tin.it

Received February 01, 2017; Accepted February 23, 2017; Published February 27, 2017

Citation: Anticoli S, Bravi MC, Siniscalchi A, Pezzella FR (2017) Rotigotine as Pharmacological Augmentation Strategy of Early Rehabilitation of Acute Ischemic Stroke Patient. J Bioanal Biomed 9: 072-072. doi:10.4172/1948-593X.1000156

Copyright: ๑ 2017 Anticoli S, et al. This is an open-access article distributed under the terms of the Creative Commons Attribution License, which permits unrestricted use, distribution, and reproduction in any medium, provided the original author and source are credited. 